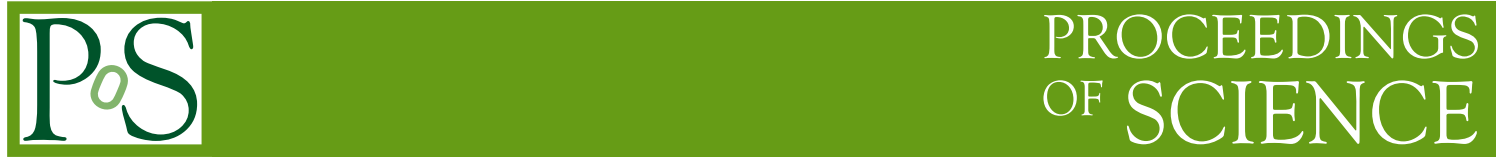

\title{
Partial quenching and chiral symmetry breaking
}

\author{
Michael Creutz* \\ Brookhaven National Laboratory \\ E-mail: mike@latticeguy. net
}

Partially quenched chiral perturbation theory assumes that valence quarks propagating on gauge configurations prepared with sea quarks of different masses will form a chiral condensate as the valence quark mass goes to zero. I present a counterexample involving non-degenerate sea quarks where the valence condensate does not form.

The 32nd International Symposium on Lattice Field Theory

23-28 June, 2014

Columbia University New York, NY

\footnotetext{
* Speaker.
} 


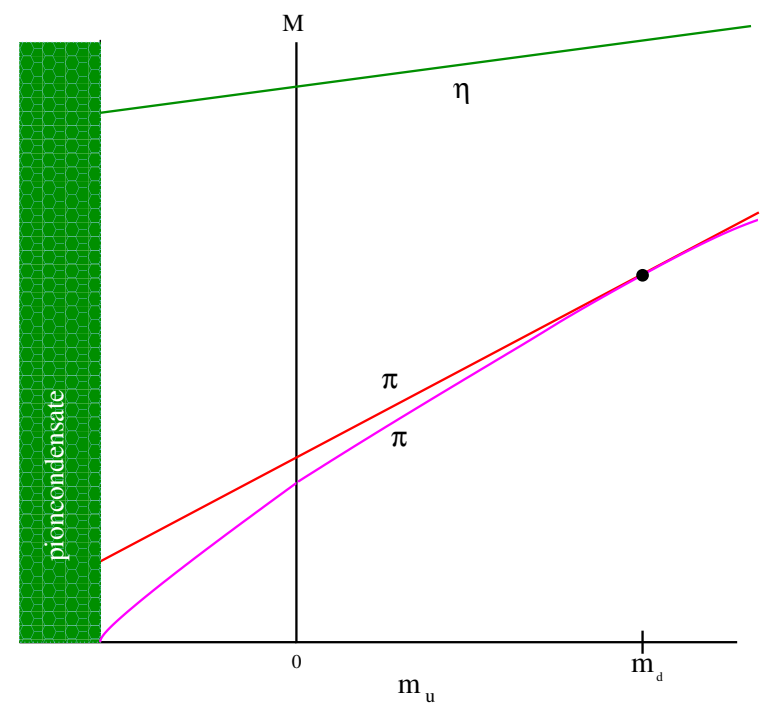

Figure 1: A schematic representation of how the pseudo-scalar masses depend on the up quark mass with a fixed non-vanishing down quark mass. The theory maintains a mass gap even at vanishing up quark mass.

Partial quenching is a common approximation used to extract additional information from sets of field configurations obtained in dynamical lattice gauge simulations. The idea is to take these configurations, generated with some set of fixed "sea" quark masses, and then study quark propagators using different "valence" quark masses. From these propagators, one then constructs "valence" bound states and studies their properties. Of course when the sea and valence masses are equal, this is just the normal lattice prescription for obtaining hadronic properties.

The conventional assumption is that, as the valence masses go to zero, a valence quark condensate will form on which one might expect the valence pion masses to go to zero with the square root of the valence quark mass. The point I wish to make is that in some cases this assumption can fail. I will show an example, using two non-degenerate sea quark masses, where the valence pions do not become massless in the valence chiral limit.

Consider two non-degenerate quark flavors to which I give the conventional names " $u$ " and " $d$." The standard chiral symmetry prediction for the dynamical pions is that their mass is controlled by the average quark mass

$$
M_{\pi}^{2} \sim \frac{m_{u}+m_{d}}{2}+O\left(m_{q}^{2}\right)
$$

Now consider fixing the down quark mass to some non-vanishing value, $m_{d} \neq 0$, and take the up quark mass to zero. Then we expect the pion mass to remain finite

$$
M_{\pi}^{2} \sim \frac{m_{d}}{2}+O\left(m_{q}^{2}\right)
$$

In particular we expect no singularity in physics for $m_{u}$ in the vicinity of zero.

Without a singularity at vanishing up quark mass, it is natural to imagine continuing the up quark mass to negative values. In standard chiral perturbation theory the neutral pion mass continues to drop until at some point it becomes negative. At this location a second order transition is expected into a $\mathrm{CP}$ violating phase with an expectation value for the neutral pion. This phase was anticipated by Dashen some time ago [1]. For a recent discussions of these issues see 
Refs. $[2,3,4,5]$. The qualitative meson mass spectrum as a function of the up quark mass is sketched qualitatively in Fig. (1).

Considerable insight into the nature of the chiral limit in QCD was provided some time ago by Banks and Casher [6]. They argued that a finite density of small eigenvalues for the Dirac operator could generate a jump in the condensate $\bar{\psi} \psi$ as the quark mass passes through zero. This is exactly as expected from chiral perturbation theory with degenerate quarks.

The situation with a single massless quark, however, generates a conundrum for this picture. As discussed above, as the sea up-quark mass passes through zero, no singularity is expected. In particular, there should be no jump in the up quark condensate $\langle\bar{u} u\rangle$ if the down quark mass remains finite. Following the Banks Casher argument, the density of up quark eigenvalues $\rho_{u}(\lambda)$ must vanish at $\lambda=0$. For another discussion of this, see Ref. [7].

Now we bring in two degenerate valence quarks and consider taking $m_{v a l}$ to zero while the dynamical up quark mass is maintained to vanish. In the limit that the valence quarks and the up sea-quark have the same mass, their propagators become identical.

$$
D_{v a l} \rightarrow D_{u} \quad \text { as } \quad m_{v a l} \rightarrow 0 .
$$

We conclude that with a vanishing dynamical up quark mass we must have

$$
\rho_{\text {val }}(0) \rightarrow \rho_{u}(0)=0 \text {. }
$$

Thus the valence quarks can not condense, there is no valence chiral symmetry breaking, and there is no expectation for the valence pion mass to go to zero. This is in direct contradiction to the usual assumptions of partially quenched chiral perturbation theory.

It is well known that in the fully quenched case, i.e. with no dynamical quarks, problems arise when the masses for valence quarks are taken to zero. In general, configurations will be encountered where the Dirac operator is not invertible and the propagators do not exist. In the example presented above, the dynamical quarks suppress such configurations so the propagators do exist, although their chiral properties are strongly modified.

While this is the basic result, some technical comments are perhaps in order. The discussion above is based on the expectation that the four dimensional free energy density behaves smoothly as the up quark mass passes through zero. The full partition function is the exponential of the volume times this density, and should be well behaved throughout the small mass region, including negative mass.

There are some peculiarities of the path integral at negative quark mass. In this situation gauge field configurations can appear for which the fermion determinant is negative. This is somewhat non-intuitive; for instance the topological susceptibility, despite being the expectation of a square, is itself negative [8]. Indeed, the susceptibility diverges to negative infinity as the Dashen phase is approached.

It should be noted that the expectation $\langle\bar{\psi} \psi\rangle$ for the up quark does not vanish at zero mass. This does not come from spontaneous chiral symmetry but rather is a direct consequence of the chiral anomaly. As discussed in [7] and [9], this expectation arises from a cancellation of the mass suppression of unit topology with an inverse mass dependence in this particular observable. As such it is tied not to a density of small eigenvalues, but rather to exact zero modes of the Dirac operator. 
This effect is only present when a single quark becomes massless; with degenerate light quarks further factors of the mass suppress topology and the Banks-Casher picture becomes relevant.

At this meeting, Verbaarschot and Wettig [10] have suggested that it might be possible for the eigenvalue density at the origin to remain finite if there is a cancellation at negative mass between configurations of non-trivial topology. However, other than the above contribution to $\langle\bar{\psi} \psi\rangle$ from unit topology, effects of higher winding number sectors are suppressed by powers of the quark mass. Also, for local observables, at large volume one can avoid these issues by working in the zero winding number sector [11].

In summary, I have presented a situation where partially quenched chiral perturbation theory can fail. One should be particularly suspicious of the approach whenever the valence quark masses become small compared to the average sea quark mass. This conclusion is a direct consequence of the anomaly and applies for any valid lattice fermion formulation.

\section{References}

[1] Roger F. Dashen. Some features of chiral symmetry breaking. Phys.Rev., D3:1879-1889, 1971.

[2] M. Creutz, "Chiral symmetry and lattice fermions," PoS QCD -TNT-III, 009 (2014) [arXiv:1309.6288 [hep-lat]].

[3] S. Aoki and M. Creutz, "Pion masses in 2-flavor QCD with $\eta$ condensation," Phys. Rev. Lett. 112, 141603 (2014) [arXiv:1402.1837 [hep-lat]].

[4] S. Aoki and M. Creutz, contribution to this meeting (2014).

[5] D. Horkel, contribution to this meeting (2014).

[6] Tom Banks and A. Casher. Chiral symmetry breaking in confining theories. Nucl.Phys., B169:103, 1980.

[7] M. Creutz, "Fun with Dirac eigenvalues," hep-lat/0511052.

[8] M. Creutz, "Quark masses, the Dashen phase, and gauge field topology," Annals Phys. 339, 560 (2013) [arXiv:1306.1245 [hep-lat]].

[9] M. Creutz, “One flavor QCD,” Annals Phys. 322, 1518 (2007) [hep-th/0609187].

[10] J. Verbaarschot and T. Wettig, contribution to this meeting (2014).

[11] S. Aoki, H. Fukaya, S. Hashimoto and T. Onogi, "Finite volume QCD at fixed topological charge," Phys. Rev. D 76, 054508 (2007) [arXiv:0707.0396 [hep-lat]]. 